\title{
Self-avoiding walks in slits and slabs with interactive walls
}

\author{
A. L. Owczarek • R. Brak • A. Rechnitzer
}

Received: 10 August 2007 / Accepted: 21 November 2007

(C) Springer Science+Business Media, LLC 2008

\begin{abstract}
Self-avoiding walk models of a polymer confined between two parallel attractive walls in two and three dimensions (slits and slabs, respectively) have recently had a revival of interest. They were first studied as simple models of steric stabilisation and sensitised flocculation in colloids. The revival has been catalysed by new exact solution techniques, that have allowed the solution of directed walk models in two dimensions in full generality, and by new Monte Carlo techniques that have allowed the simulation of the full parameter space in the three-dimensional slab model. Additionally, rigorous techniques applied to the slab problem have also yielded new results. The contributions to the study of this problem that have been recently added include a novel phase diagram for the "infinite-slab" (when the walls are a macroscopic distance apart but both walls may still "see" the polymer) the delineation of the repulsive and attractive regimes of the parameter space, and a conjectured scaling theory for the problem in general dimensions.
\end{abstract}

Keywords Self-avoiding walks $\cdot$ Slab $\cdot$ Slit $\cdot$ Adsorption $\cdot$ Lattice polymers $\cdot$ Steric stabilisation

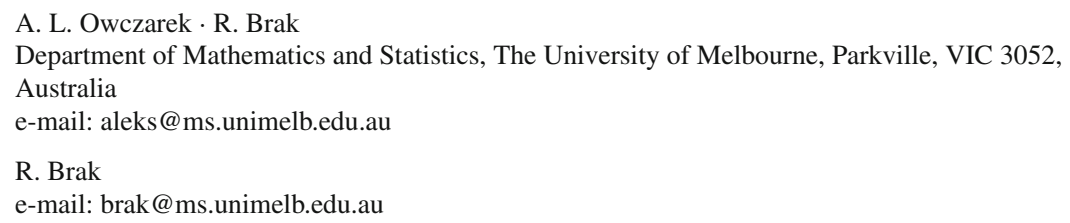




\section{Introduction}

Self-avoiding walks are a common model of flexible polymers in solution [1]. Confining self-avoiding walks between two parallel walls where the walks interact with the walls can be considered a simple model of the steric stabilisation and sensitised flocculation of colloidal dispersions [2] by polymers. Both the two-dimensional case of a polymer in a slit and the three-dimensional case of a polymer in a slab have been studied. The seminal work of DiMarzio and Rubin [3] sparked the study of these problems. However, quite a bit of the work completed was concerned with the case of non-interacting walls [4-7]. The work of Daoud and de Gennes [8] is also relevant as they developed scaling laws for polymers in confined spaces. When there is an energetic interaction with the walls, the cases of a single attractive wall and equal interactions with the two walls have been investigated [3,9-13]. Importantly, the full phase space when the interactions with the two walls are allowed to vary independently has not received attention until recently [14-17]. Here we review the work on the full problem described in those four papers: the solution of a two-dimensional integrable model by Brak et al. [14]; rigorous results by Janse van Rensburg et al. [16] valid in any dimension; and numerical studies and scaling conjectures concerning the three-dimensional slab problem in the two papers by Janse van Rensburg etal. [15] and Martin et al. [17].

To begin we define the full three-dimensional lattice model which is the subject of the two papers by Janse van Rensburg et al. [15] and Martin et al. [17]. Consider the simple cubic lattice with coordinate system $(x, y, z)$ so that each vertex has integer coordinates. Consider $n$-edge self-avoiding walks starting at the origin with vertices numbered $j=0,1,2, \ldots n$ and with the $j$ th vertex having integer coordinates $\left(x_{j}, y_{j}, z_{j}\right)$. We shall be interested in such walks confined so that $0 \leq z_{j} \leq w$ for fixed $w$. If we keep track of how many vertices are in each of the two planes $z=0$ and $z=w$ we can define the partition function:

$$
Z_{n}(a, b ; w)=\sum_{u \geq 0} \sum_{v \geq 0} c_{n}(u+1, v ; w) a^{u} b^{v}
$$

where $c_{n}(u+1, v ; w)$ is the number of $n$-edge self-avoiding walks, starting at the origin, confined between the two planes $z=0$ (bottom wall) and $z=w$ (top wall), with $u+1$ vertices in $z=0$ and with $v$ vertices in $z=w$. The variables $a$ and $b$ are then Boltzmann factors associated with vertices in the planes $z=0$ and $z=w$, respectively. For $a>1$ and $b>1$ an attractive potential is felt by the monomers of the our polymer, modelled by the vertices of the walk, visiting the bottom and top walls, respectively. We define the finite-size finite-slab free energy $\kappa_{n}$ as

$$
\kappa_{n}(a, b ; w)=n^{-1} \log Z_{n}(a, b ; w)
$$

and the thermodynamic limiting finite-slab free energy as

$$
\kappa(a, b ; w)=\lim _{n \rightarrow \infty} n^{-1} \log Z_{n}(a, b ; w) .
$$


As we shall see below this limit exists [16]. One may then consider the behaviour of $\kappa(a, b ; w)$ in the limit as the width tends to infinity: we shall refer to this limit as the infinite slab limit and define the infinite slab free energy, $\kappa(a, b)$, as the point-wise limit

$$
\kappa(a, b)=\lim _{w \rightarrow \infty} \kappa(a, b ; w) .
$$

One can then explore the location of singularities in $\kappa(a, b)$ and it is the loci of these singularities that determine the phase diagram of the infinite-slab in the $(a, b)$-plane. Physically, taking the polymer length limit first means that we are investigating the thermodynamics of macroscopic polymers in slabs of mesoscopic widths.

The $w$-dependence of $\kappa(a, b ; w)$ determines if the force exerted by the walk on the confining walls is repulsive or attractive. The force at finite polymer length is defined as

$$
F_{n}(a, b ; w)=\kappa_{n}(a, b ; w)-\kappa_{n}(a, b ; w-1)
$$

while the thermodynamic limit is

$$
F(a, b ; w)=\lim _{n \rightarrow \infty} F_{n}(a, b ; w)=\kappa(a, b ; w)-\kappa(a, b ; w-1) .
$$

Hence if $\kappa(a, b ; w)$ is an increasing function of $w$ the force is repulsive while if it is a decreasing function of $w$ the force is attractive. The scaling theory of Daoud and de Gennes [8] predicts that when $a=b=1$

$$
F(a, b ; w) \sim \frac{C}{w^{(1+1 / v)}} \quad \text { as } w \rightarrow \infty
$$

where $v$ is the radius of gyration exponent for self-avoiding walks in three dimensions.

There is a second double limit of the finite free energy, $\kappa_{n}(a, b ; w)$, where the length and width limits are exchanged. This more traditional case gives us the halfspace model which describes the adsorption of polymers on a single wall. Since we are considering self-avoiding walks that are attached to the surface where the sites are weighted with the Boltzmann weight $a$ the limit of large width for fixed length is independent of the Boltzmann weight $b$ : the partition function then simply becomes that of a self-avoiding walk attached to the surface in a half-space. Hence if we define

$$
\bar{\kappa}_{n}(a)=\lim _{w \rightarrow \infty} n^{-1} \log Z_{n}(a, b ; w)
$$

then the infinite length limit of $\bar{\kappa}_{n}(a)$, that is,

$$
\bar{\kappa}(a)=\lim _{n \rightarrow \infty} \bar{\kappa}_{n}(a),
$$

gives us the thermodynamic free energy, $\bar{\kappa}(a)$, of the half-space. This free energy $\bar{\kappa}(a)$ has a single singularity at an adsorption transition $a=a_{c}$ [18], where the current 
best estimate is $a_{c} \approx 1.33$ [19]. In fact, it has been previously proved [18] that $\bar{\kappa}(a)$ is constant for $a \leq a_{c}$ and is given by the value $\log \mu_{3}$, that is, the logarithm of the growth constant for unconfined self-avoiding walks in three dimensions. This adsorption transition can be characterised by considering the density of visits to the wall, $\rho(a)$, where

$$
\rho(a)=\lim _{n \rightarrow \infty} \frac{\langle u\rangle}{n}
$$

which acts as an order parameter for the phase transition. For $a \leq a_{c}$ we have that $\rho=0$ while for $a>a_{c}$ we have $\rho>0$, and $\rho \rightarrow 0$ as $a \rightarrow a_{c}^{+}$as the transition is a second-order phase transition. It is therefore a central question to compare the two double limits of the half-space $\bar{\kappa}(a)$ to the infinite slit $\kappa(a, b)$ : this is precisely what has been done in the four papers reviewed herein.

The standard scaling hypothesis for self-avoiding walks attached to a surface [20] in the half-space predicts

$$
\bar{\kappa}_{n}(a) \sim \bar{\kappa}(a)+\left(\gamma_{1}-1\right) \frac{\log n}{n}+\frac{A(a)}{n} \quad \text { as } n \rightarrow \infty .
$$

The (universal) value of $\gamma_{1}$ depends on whether $a<a_{c}, a=a_{c}$, or $a>a_{c}$ [20]. For $a<a_{c}$ the exponent $\gamma_{1}$ has been estimated as 0.68 [20]. For $a=a_{c}$ the exponent $\gamma_{1}$ is often denoted by $\gamma_{1, s}$. Previously, it was known imprecisely as 1.5(2) [20], and the value used in the scaling plots of Martin etal. [17] found that 1.25 was an effective estimate. For $a>a_{c}$ the half-space exponent $\gamma_{1}$ in three dimensions takes on the "bulk" two-dimensional value 43/32 [21]. In a slab of any finite width one expects two-dimensional behaviour to eventuate so that

$$
\kappa_{n}(a, b ; w) \sim \kappa(a, b ; w)+(\gamma-1) \frac{\log n}{n}+\frac{B(a, b ; w)}{n} \quad \text { as } n \rightarrow \infty,
$$

where once again $\gamma=43 / 32$ regardless of $a$ and $b$. The question then arises as to the scaling form in the two variables polymer length, $n$, and slab width, $w$, for $\kappa_{n}(a, b ; w)$ : this was discussed by Martin et al. [17] and we summarise their conjectures below.

We continue by discussing the exact results of Brak et al. [14] on a two-dimensional integrable model in the next section. It was this work that motivated the renewed interest in the three-dimensional slab problem, and it proves remarkable, at least on first sight, how many of the features of the exact solution have shown up in the analysis of the three-dimensional model. After that we discuss the rigorous results, derived in arbitrary dimension, concerning the existence and analyticity properties of the limiting free energy found Janse van Rensburg et al. [16] before moving onto to discussing the numerical results on the phase and force diagrams for the infinite slab as well as the scaling theory for finite slabs. We end with a brief summary. 


\section{Exact solution of the directed model in a slit}

In this section we review the main results of an exactly solved model of directed paths in a slit of width $w$. While these results appear in [14], we will use a different combinatorial construction to derive them. In particular, a Temperley-like argument gives a functional equation which we will then solve using the kernel method (see [22] and references therein). Importantly, the results summarised below provide the impetus for the work on undirected walks in three-dimensional slabs, described in the next section.

Consider paths on $\mathbb{Z}^{2}$ confined to the strip $0 \leq y \leq w$ that start at $(0,0)$, and take steps $(1, \pm 1)$. We define a generating function for these walks by

$$
f(s ; z ; a, b)=\sum_{\varphi} s^{h(\varphi)} z^{|\varphi|} a^{v_{l}(\varphi)} b^{v_{u}(\varphi)}=\sum_{k=0}^{w} f_{k}(z ; a, b) s^{k},
$$

where the first sum ranges over all directed walks confined to the slit, $z$ is conjugate to the length of walk, $a, b$ are conjugate to the number of visits to the top and bottom walls of the slit and $s$ is conjugate to the height of the final vertex. The function $f_{k}$ is the generating function of the subset of walks that end at height $k$. In the analysis that follows we will primarily concern ourselves with loops which are walks that end at height 0 and so are counted by $L_{w}(z ; a, b) \equiv f_{0}(z ; a, b)=f(0 ; z, a, b)$. One can show that fixing the height of the last vertex of the walk does not change the thermodynamic $(n \rightarrow \infty)$ free-energy of the system.

We compute $f(s ; z ; a, b) \equiv f(s)$ by constructing walks a single step at a time; every directed walk in the strip is either a single vertex or can be obtained by adding a step (in the $(1, \pm 1)$ directions) to a shorter walk. When adding steps to a shorter walk we have to exclude those configurations which step outside the strip-see Fig. 1. We define the notation $\bar{s}=1 / s$. The single vertex contributes 1 to the generating function. Adding a single step to an existing walk contributes $z(s+\bar{s}) f(s)$, however this includes some configurations that leave the strip. A walk that takes a single step below the line $y=0$ contributes $z \bar{s} f_{0}$ and one that steps above the line $y=w$ is given by $z s^{w+1} f_{w}$. This leads to the equation

$$
f(s)=1+z(s+\bar{s}) f(s)-z f_{0}-z s^{w} f_{w},
$$

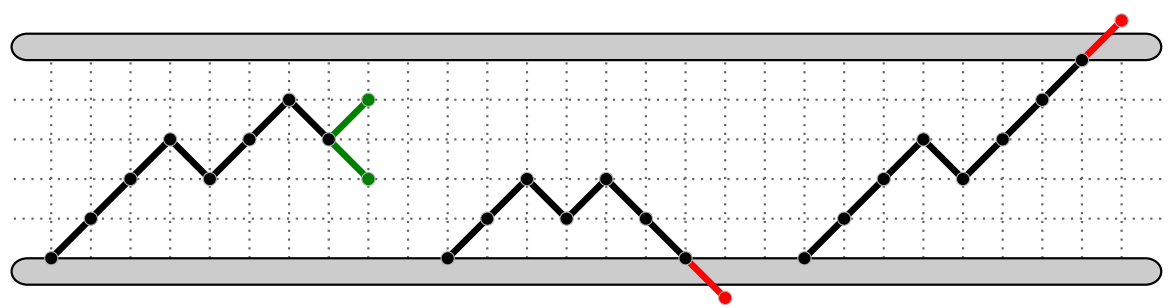

Fig. 1 Every walk is either a single vertex or can be obtained by adding a $(1, \pm 1)$ step to a shorter walk. When appending these steps one must remove the contribution from the walks that step outside the strip 
valid for $a=b=1$. However, this does not take into account the newly generated visits to either wall. A walk that steps down onto the bottom wall is counted by $z a f_{1}$ (since it steps from height 1 to height 0 ) and similarly a walk that steps up to the top wall is counted by $z b f_{w-1}$. These walks have already been counted by the $z(s+\bar{s}) f(s)$ term but without the weights $a$ and $b$, so we must remove the incorrectly weighted contributions and then replace them by the correct weight. This gives

$$
f(s)=1+z(s+\bar{s}) f(s)-z f_{0}-z s^{w} f_{w}+z(a-1) f_{1}+z s^{w}(b-1) f_{w-1} .
$$

This is a single equation in 5 "unknowns". We can remove two of these unknowns by examining the coefficients of $s^{0}$ and $s^{w}$ :

$$
\begin{aligned}
& f_{0}=1+z f_{1}+z(a-1) f_{1}=1+z a f_{1}, \\
& f_{w}=z f_{w-1}+z(b-1) f_{w-1}=z b f_{w-1} .
\end{aligned}
$$

Rewriting the main equation in terms of $f(s), f_{0}$ and $f_{w}$ gives

$$
\left[1-z\left(s+\frac{1}{s}\right)\right] f(s)=\frac{1}{a}+\left(1-\frac{1}{a}-z \bar{s}\right) f_{0}+\left(1-\frac{1}{b}-z s\right) s^{w} f_{w}
$$

The coefficient of $f(s)$ in the above equation is called the kernel. We proceed by substituting values of $s$ that set the kernel to zero:

$$
s=\sigma_{ \pm}=\frac{1 \pm \sqrt{1-4 z^{2}}}{2 z}
$$

We note that $\sigma_{-}=z+O\left(z^{3}\right)$ and $\sigma_{+}=z^{-1}+O(z)$ and that $\sigma_{+} \sigma_{-}=1$. Typically when applying the kernel method, one would only make use of those kernel roots that are formal power series such as $\sigma_{-}$. However, we note that since the coefficient of $z^{n}$ is a polynomial in $s$ of degree $\min \{n, w\}$, substituting $s=\sigma_{+}$also results in formal power series. This gives two linear equations involving the unknowns $f_{0}$ and $f_{w}$ :

$$
0=\frac{1}{a}+\left(1-\frac{1}{a}-\frac{z}{\sigma_{ \pm}}\right) f_{0}+\left(1-\frac{1}{b}-z \sigma_{ \pm}\right) \sigma_{ \pm}^{w} f_{w}
$$

Solving these gives

$$
\begin{aligned}
& L_{w}(z ; a, b) \equiv f_{0} \\
& \quad=\frac{\left(\sigma_{-}^{2}+1\right)\left(\left(\sigma_{-}^{2}+1-b\right) \sigma_{-}^{2 w}+\left(\sigma_{-}^{2} b-\sigma_{-}^{2}-1\right)\right)}{\left(\sigma_{-}^{2}+1-b\right)\left(\sigma_{-}^{2}-a+1\right) \sigma_{-}^{2 w}-\left(\sigma_{-}^{2} b-\sigma_{-}^{2}-1\right)\left(a \sigma_{-}^{2}-\sigma_{-}^{2}-1\right)}
\end{aligned}
$$

and a similar expression for $f_{w}$. We have used the fact that $\sigma_{+} \sigma_{-}=1$ and $1=$ $z\left(\sigma_{-}+\sigma_{-}^{-1}\right)$. Further, if we write $q=\sigma_{-}^{2}$ then we recover Eq. 5.7 from [14].

The dominant singularity of $L_{w}$ determines the free-energy of the underlying physical system. The singularities of $f_{0}$ are the zeros of its denominator and, possibly, the 
square-root singularity of $\sigma_{-}$at $z=1 / 2$. It can be proved that $f_{0}$ is rational in $z$ and hence has no algebraic singularities, only poles. Hence the singularities in $z$ (and so $q$ ) are poles. The zeros of the denominator are given by the solutions of

$$
q^{w}=\frac{(b q-q-1)(a q-q-1)}{(q-b+1)(q-a+1)},
$$

which we note is symmetric in $a \leftrightarrow b$. Note also that if one solves the problem where walks end at some general height then exactly the same singularities appear-hence all these problems have the same thermodynamic free-energy.

The expression for $L_{w}$ simplifies considerably at the points $a, b \in\{1,2\}$ and along the curve $a b=a+b$ (see [14]), and at these points one is able to find the dominant singularity, and so the free-energy, in closed form:

$$
\begin{aligned}
\kappa(1,1 ; w) & =\log (2 \cos (2 \pi /(w+2))) ; \\
\kappa(1,2 ; w)=\kappa(2,1 ; w) & =\log (2 \cos (\pi /(w+1))) ; \\
\kappa(2,2 ; w) & =\log 2 ;
\end{aligned}
$$

and along the curve $a b=a+b$

$$
\kappa^{\text {curve }}(w)= \begin{cases}\log \left(\frac{a}{\sqrt{a-1}}\right) & \text { for } a \geq b \\ \log \left(\frac{b}{\sqrt{b-1}}\right) & \text { for } a<b .\end{cases}
$$

It is important to note that these last two expressions for the free-energy are independent of $w$. This implies that along the curve $a b=a+b$ (which includes the point $(2,2))$ the thermodynamic limit of the system is independent of the width of the strip.

Turning to general $(a, b)$, Brak et al. [14] were unable to find closed form expressions for the solutions of Eq. 2.21 but they were able to find asymptotic expansions for large $w$ by perturbing around the exactly solved points in descending powers of $w$. This gives

$$
\begin{aligned}
\kappa(a, b ; w)= & \log (2)-\frac{\pi^{2}}{2 w^{2}}+O\left(w^{-3}\right) \text { for } a, b<2, \\
\kappa(a, 2 ; w)= & \log (2)-\frac{\pi^{2}}{8 w^{2}}+O\left(w^{-3}\right) \text { for } a<2, \\
\kappa(a, b ; w)= & \log \left(\frac{a}{\sqrt{a-1}}\right)+\frac{(a-2)^{2}(a b-a-b)}{2(a-1)(a-b)}\left(\frac{1}{a-1}\right)^{w} \\
& +O\left(\frac{w}{(a-1)^{2 w}}\right) \text { for } a>\max \{b, 2\} \\
\kappa(a, a ; w)= & \log \left(\frac{a}{\sqrt{a-1}}\right)+\frac{(a-2)^{2}}{2(a-1)}\left(\frac{1}{a-1}\right)^{w / 2} \\
& +O\left(\frac{w}{(a-1)^{w}}\right) \text { for } a=b>2
\end{aligned}
$$


and the relations obtained by the manifest $a \leftrightarrow b$ symmetry of the problem in the large length limit, as noted above in the symmetry of Eq. 2.21.

As explained in the introduction, taking the limit of these expressions as $w \rightarrow \infty$ one arrives at the infinite-slit limit (in which we have let the length of the polymer go to infinity before the width of the slit). The free energy is then given by

$$
\kappa(a, b)= \begin{cases}\log 2 & \text { for } a, b \leq 2, \\ \log \left(\frac{a}{\sqrt{a-1}}\right) & \text { for } a>\max \{b, 2\}, \\ \log \left(\frac{b}{\sqrt{b-1}}\right) & \text { otherwise. }\end{cases}
$$

On the other hand, if we let $w \rightarrow \infty$ before we let the length of the polymer go to infinity, then we effectively remove the top wall (see above). This leads to the following functional equation satisfied by the generating function:

$$
f(s)=1+z(s+\bar{s}) f(s)-z \bar{s} f_{0}+z(a-1) f_{1} .
$$

Repeating the kernel method one can obtain the generating function, $L(z, a)$, of directed paths that start and end on the line $y=0$ and interacting with that line:

$$
L(z, a)=\frac{1+\sigma_{-}^{2}}{1+\sigma_{-}-a \sigma_{-}} .
$$

Note that this is independent of $b$. This function has two singularities: the square-root singularity of $\sigma_{-}$at $z=1 / 2$ and a simple pole at the zero of the denominator. The free-energy is then

$$
\bar{\kappa}(a)= \begin{cases}\log 2 & \text { for } a \leq 2 \\ \log \left(\frac{a}{\sqrt{a-1}}\right) & \text { for } a>2\end{cases}
$$

The expression (2.15) shows there is a single second-order phase transition at $a=2$ with a jump in the second-derivative with respect to the fugacity $a$ on crossing the transition (i.e. a jump in the specific heat). It is an adsorption transition where the polymer has a zero thermodynamic density of visits to the wall, $\rho(a)$, for $a<2$ (calculated from a the first derivative of the free energy with respect to $a$ ) and a finite density for $a>2$. The expression (2.12) for the infinite-slit, and its derivatives by $a$ and $b$, giving the density of visits to each of the walls, imply that for $a, b<2$ the polymer is desorbed from both walls. For $a>\max \{2, b\}$ the polymer is adsorbed to the bottom surface and for $b>\max \{a, 2\}$ the polymer is adsorbed to the top surface. The two transitions from desorbed to adsorbed on either wall are both second-order transitions of identical type to the half-plane desorption transition. However, there is also a first-order transition line between the two adsorbed phases along the line $a=b$ for $a, b>2$. We plot the phase diagrams implied by these two different free-energies (Eqs. 2.27 and 2.24) in Fig. 2. It is clear that the order in which the polymer length 

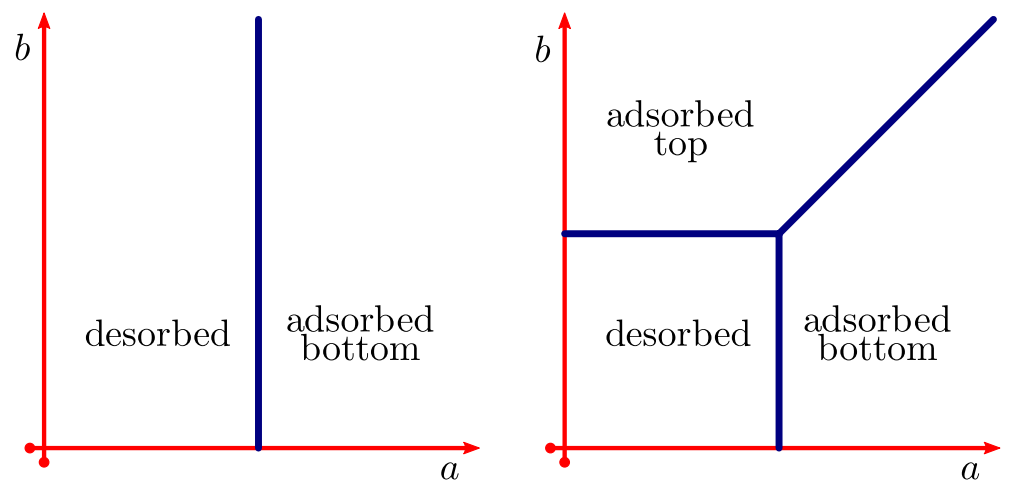

Fig. 2 The half-plane and infinite-slit phase diagrams. In the half-plane limit, there are only two phases separated by a second-order adsorption transition. There are three phases in the infinite-slit limit: a desorbed phase and two adsorbed phases, one adsorbed to the bottom wall and the other adsorbed to the top wall. The transition between the two adsorbed phases is first-order

and strip width are taken to infinity produces a large change in the behaviour of the system. In particular, the two limits do not commute.

Differentiating with respect to $w$ one obtains expressions for the force exerted by the polymer on the walls of the strip. These expressions imply three distinct regions in which the system exhibits different behaviour. For $a, b \leq 2$ the force is repulsive and decays as $w^{-3}$ - we interpret this as a long-range force. Outside this square, the force decays exponentially with $w$-which we interpret as a short range force. Furthermore, the sign of the force changes as the curve $a b=a+b$ is crossed. In particular, to the left of this curve the force is repulsive, on this curve the force is identically zero (since the free-energy is independent of $w$, as noted above) and to the right of this curve the force is attractive. We also note that along the line $a=b>2$ the force decays more slowly than elsewhere in the repulsive region (Fig. 3).

\section{Self-avoiding walks in interactive slabs}

\subsection{Rigorous results for general dimensions}

Janse van Rensburg et al. [16] developed new pattern-type theorems for walks on a $d$-dimensional hyper-cubic lattice confined between two $(d-1)$-dimensional planes. Here the dimension $d \geq 2$. They used these and concatenation arguments to prove several results concerning such systems. They proved that:

- the $d$-dimensional analogue of $\kappa(a, b ; w)$ exists for all $a$ and $b$;

- $\kappa(1,1 ; w)=\kappa_{S A W}$ where $\kappa_{S A W}$ is the bulk connective constant;

- $\kappa(a, 1 ; w)$ is a convex function of $\log a$, and so is continuous for $a>0$. It is also differentiable almost everywhere in $a$ for $a>0$;

- $\kappa(a, 1)=\bar{\kappa}(a)$

- $\kappa(a, a)=\bar{\kappa}(a)=\kappa_{S A W}=$ constant for $a<1$;

- $\kappa(a, b)=\bar{\kappa}(a)$ if $b \leq 1$ and $\kappa(a, b)=\bar{\kappa}(b)$ if $a \leq 1$. 
Fig. 3 A diagram of the different regions of force exerted by the polymer on the walls of the strip. The long-range behaviour refers to a power-law decay, while the short-range behaviour indicates an exponential decay. The zero-force line is given by $a b=a+b$. Along the dashed line $a=b$ there is a change in the nature of the repulsive force

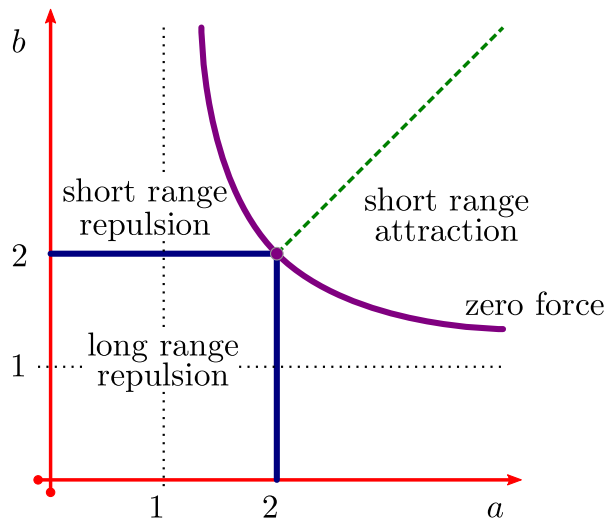

These results and some other suggestive inequalities point to a phase diagram qualitatively the same as that of the directed walk case explored above. They also consequently showed that for $b<1$ and $a<1$ that $F(a, b ; w)$ is positive and so repulsive for all $w$.

\subsection{Phase diagram for the slab $(d=3)$}

Monte Carlo data for walks up to length 128 and widths up to 11 was considered by Janse van Rensburg et al. [15]. In particular the fluctuations in the numbers of visits to the wall, that is in $\langle u\rangle$ and $\langle v\rangle$, is reproduced in Fig. 4, as a function of $a$ and $b$. The results show strong fluctuations for $a=a_{c}, b<b_{c}$, for $b=b_{c}, a<a_{c}$ and for $b=a, a>a_{c}$. Also, $b_{c} \approx a_{c}$. These were interpreted as finite $w$ remnants of lines of phase transitions in the $w \rightarrow \infty$ limit, corresponding to adsorption transitions on the two walls, and to a transition from adsorption on one wall to adsorption on the other wall as we cross the line $b=a$. It was concluded that symmetry implies $b_{c}=a_{c}$. This is exactly the same as one sees in the integrable model above given the caveat that $a_{c}$ takes on a different value. Given the similarity to the integrable case the interpretation of this data is as follows. There exists a transition value equal to the half-space adsorption point $a_{c}=b_{c} \approx 1.33$ such that for $a$ and $b$ below this critical point the polymer is desorbed from both walls. For $a>\max \left\{a_{c}, b\right\}$ the polymer is adsorbed to the bottom surface and for $b>\max \left\{a, a_{c}\right\}$ the polymer is adsorbed to the top surface. Hence, there is a transition line between the two adsorbed phases along the line $a=b$ for $a, b>a_{c}$. It remained to be seen whether the transition for $a=a_{c}$, $b<a_{c}$ and $b=a_{c}, a<a_{c}$ are second-order and exactly of the same type as the half-space adsorption while the transition along the line $b=a, a>a_{c}$ is first-order as in the integrable model discussed above.

Martin et al. [17] investigated these questions using Monte Carlo simulations with walks up to length 512 and slab widths up to 40 lattice spaces. They checked that the scaling of the peaks of the fluctuations did indeed demonstrate phase transitions in the thermodynamic and large width limits. Given the expected infinite-slab phase diagram, which is displayed in Fig. 5, four lines were analysed in detail: $(1 / 2, b),(2, b),(a, 1 / 2)$ and $(a, 2)$. The results confirmed the expected phase diagram 


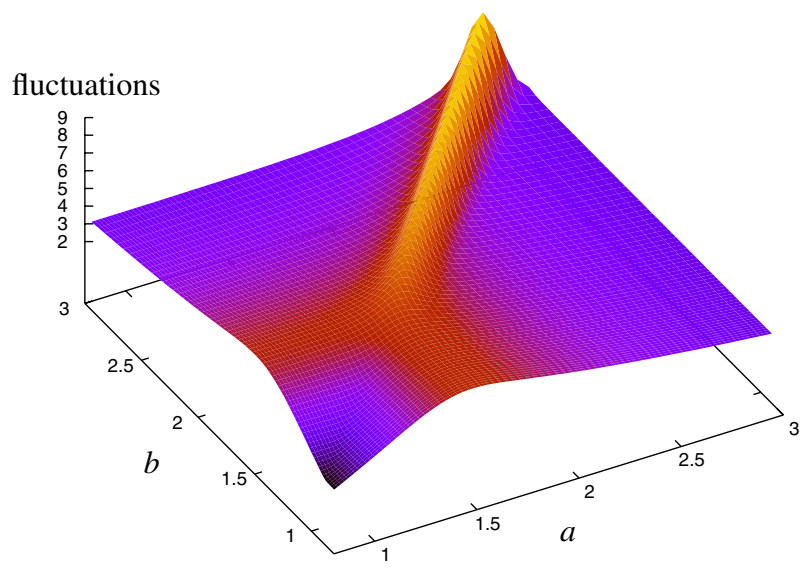

Fig. 4 The largest eigenvalue of the matrix of fluctuations in the numbers of visits to the confining walls for the simple cubic lattice as a function of $a$ and $b$

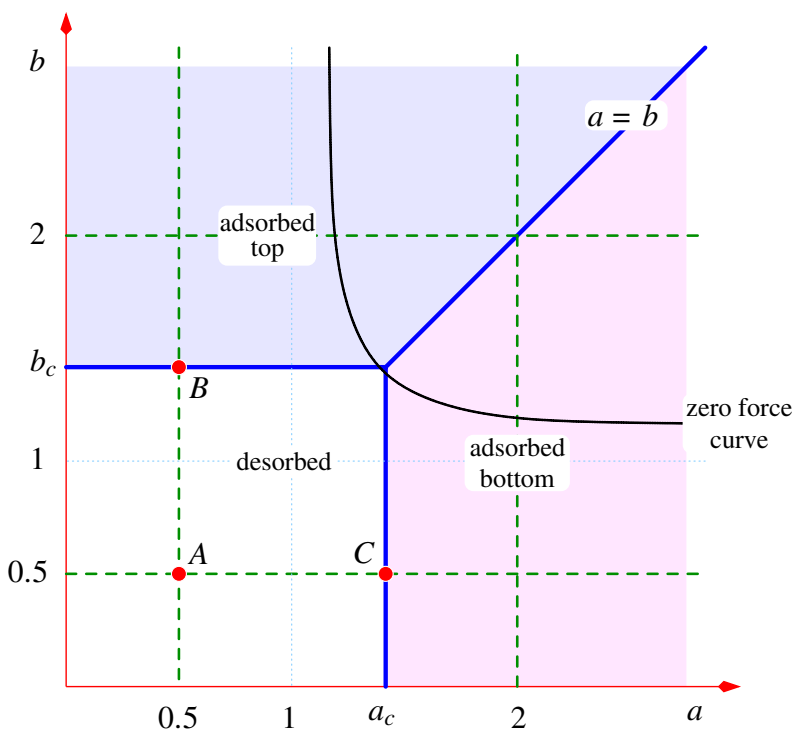

Fig. 5 The conjectured infinite-slab phase diagram contains three phases in which the polymer is desorbed, adsorbed to the bottom surface and adsorbed to the top surface. The corresponding phase boundaries are indicated with solid lines. The system was simulated along the lines $\{(a, 1 / 2),(a, 2),(1 / 2, b),(2, b)\}$ by Martin et al. [17] (indicated with dashed lines). The three points A, B and C are those at which we estimate the scaling function

in Fig. 5 and transition types as described. In particular, they found that the transitions on the lines $(a, 1 / 2)$ and $(1 / 2, b)$ occurred at $(1.38(4), 1 / 2)$ and $(1 / 2,1.38(4))$ so indeed the two transition values were the same and equal, within numerical precision, to the half-space adsorption point. Moreover, the transition was second-order with a crossover exponent near 0.5 as expected for the single wall adsorption problem. 


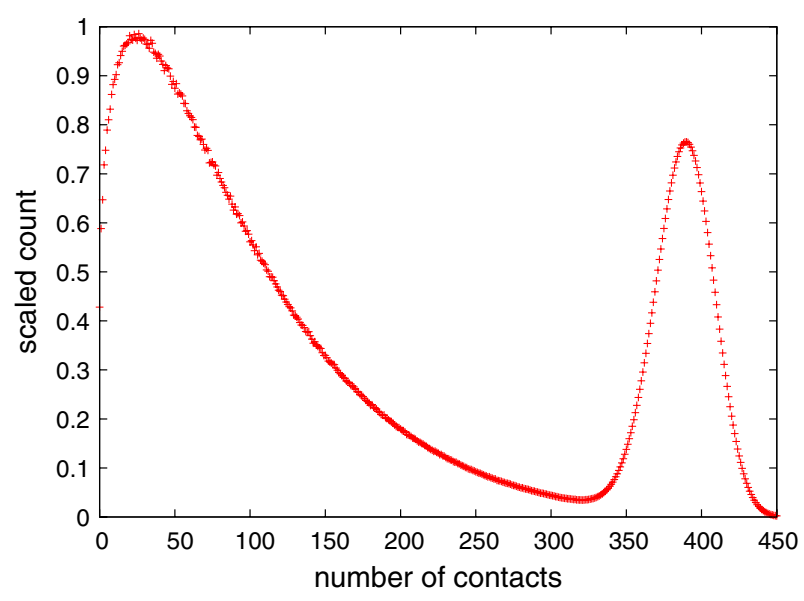

Fig. 6 The distribution of contacts with the bottom surface from the simulations along the line $(a, 2)$ where $a$ was chosen to be at the peak of the variance of contacts with the bottom surface. The value of $a$ used was 1.975, for data produced from simulations at width 12 and polymer length 512

The crossover exponent, $\phi$, is related to the specific heat exponent via the relation $\alpha=2-1 / \phi$. The lines $(a, 2)$ and $(2, b)$ yielded a transition that occurred approximately at $a=2$ and $b=2$ respectively, and as expected. Moreover, the convergence of the peak heights of the fluctuations divided by the square of the length indicate a crossover exponent of $\phi=1$, which in turn implies a specific heat exponent $\alpha=1$, that is, a first-order transition. This was confirmed by plotting the distribution of the contacts with the bottom surface at the transition: this is shown in Fig. 6, where we clearly see two peaks. Such a bimodal distribution is the hallmark of a first-order transition. Hence the phase diagram in Fig. 5, which mimics the two-dimensional directed walk phase diagram, was confirmed.

\subsection{Force diagram for the slab $(d=3)$}

The force has also been studied using both Monte Carlo and exact enumeration techniques in $[15,17]$. It was found via exact enumeration data in [15] that considering $b=1$ the force was positive (repulsive) for all $a$ and decreases as $a$ increases. The force was then was scaled with the factor $w^{1+1 / v}$, so as to check if Eq. 3.4 can be extended to other values of $a$ and $b$. One would expect that it could indeed be extended to all parts of the desorbed phase, i.e. $0 \leq a, b<2$, and, perhaps, to the phase boundaries of this region. The scaled force $w^{1+1 / v} F$ was observed to weakly collapse for $b<a_{c}$. Clearly corrections to scaling were still evident as finite length walks and small widths were used in the simulations. Using the same types of data the line $a=b$ was also examined and it was found that the force was repulsive for $a \lesssim a_{c}$ and attractive for $a>a_{c}$. Along the line $a=b$ the scaled force $w^{1+1 / v} F$ converged more quickly to a non-zero constant for $a \lesssim a_{c}$, as the width was increased, than for the line $b=1$. For $a>a_{c}$ the scaled combination did not converge. 
In [17] the regions of attractive and repulsive forces were examined using exact enumeration data for $n \leq 22$ and $w \leq 8$. Using an analysis of the ratio

$$
R_{n}(a, b ; w)=\sqrt{Z_{n}(a, b ; w) / Z_{n-2}(a, b ; w)}
$$

and the related quantity

$$
R_{n}^{\prime}(a, b ; w)=R_{n}(a, b ; w)-\sqrt{Q_{n}(a) / Q_{n-2}(a)},
$$

where $Q_{n}(a)$ is the partition function for the half-space problem the following results were found. There exists a single zero-force curve, as in Fig. 5, that is conjectured to go through the point $\left(a_{c}, a_{c}\right)$. It would seem to be asymptotic to the lines $a=1$ and $b=1$. For small $a$ and $b$ to the left of this curve the force is repulsive while to the right the force is attractive. From the data it was deduced that for $a \leq a_{c}, b \leq a_{c}$ the force is repulsive and obeys the Daoud-deGennes scaling as in Eq. 3.4. It was inferred that, as in the integrable model [14], for other values of $a$ and $b$ the force decays exponentially fast in the width. In this way the force diagram for the cubic lattice self-avoiding walk model has the same structure as the square lattice directed walk model described above. A numerical estimate of the zero-force curve was obtained in [17].

\subsection{Scaling theory in general dimensions}

In this section we summarise scaling hypotheses [17] for the free energy and the force between the walls in the high temperature and critical regimes $a, b \leq a_{c}$. Following Eq. 1.7 it is expected that for this part of the parameter space the force applied by the walk on the walls as a function of the width is expected to be a power law. It is not expected that standard scaling arguments hold in the low temperature regimes where the force is predicted to fall off exponentially with the width as mentioned above.

The fixed width scaling (1.12) can be reconciled with the infinite width scaling (1.11) using the hypothesis of a scaling function in an appropriate scaling variable. Since walks in a half-space typically extend out from the surface an amount proportional to $n^{v}$, where $v$ is the three-dimensional value of the radius of gyration exponent, one can conjecture that this scaling variable should be $n^{v} / w$. It was conjectured [17] that the scaling form of the free energy is

$$
\kappa_{n}(a, b ; w) \sim \log \mu_{3}+\left(\gamma_{1}-1\right) \frac{\log n}{n}+\frac{1}{n} \mathcal{K}\left(n^{\nu} / w\right)
$$

as $n, w \rightarrow \infty$ with $n^{v} / w$ fixed, and $\gamma_{1}$ taking on the appropriate half-space value depending on whether the value of $a$ is $a_{c}$ or less. It is important to understand that the scaling function depends on whether the underlying infinite-slit system is critical or not as the temperature is varied. Hence there are four different scaling functions: one for $a, b<a_{c}$, one for $a=a_{c}, b<a_{c}$, one for $a<a_{c}, b=a_{c}$ and one for $a=a_{c}, b=a_{c}$. 


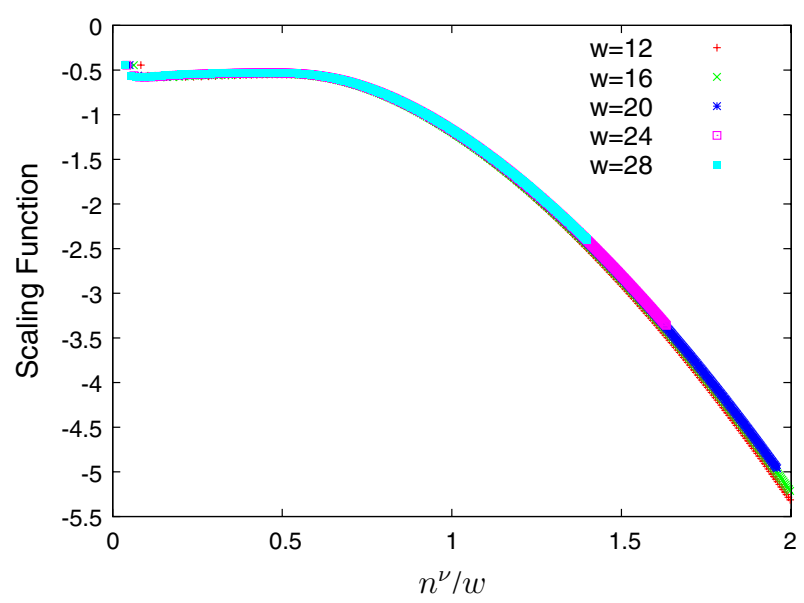

Fig. 7 A plot of the scaled free energy at the point $A(1 / 2,1 / 2)$ for widths $12,16,20,24$ and 28 and lengths from 0 to 512 . The horizontal axis is $n^{v} / w$ and the vertical axis is $n\left(\frac{\log Z_{n}(w)}{n}-\log \mu_{3}-\left(\gamma_{1}-1\right) \frac{\log n}{n}\right)$. The values $\mu_{3}=4.684, v=0.588$ and $\left(\gamma_{1}-1\right)=-0.32$ were used

The scaling of the force $F_{n}(a, b ; w)$ can be found by using

$$
\left.F_{n}(a, b ; w) \sim \frac{\partial}{\partial w} \text { (scaling form for } \kappa_{n}(a, b ; w)\right)
$$

and so it was concluded that a scaling form for the force would therefore be

$$
F_{n}(a, b ; w) \sim \frac{1}{n^{(1+v)}} \mathcal{F}\left(n^{v} / w\right) \quad \text { as } n, w \rightarrow \infty
$$

where

$$
\mathcal{F}(x) \sim c x^{1+1 / v} \quad \text { as } x \rightarrow \infty
$$

Hence the force exert by a macroscopic polymer $F(a, b ; w)$ scales as in Eq. 1.7 for all $a, b \leq a_{c}$.

To confirm the above picture the scaling function of the free energy was studied at three points in the $(a, b)$-plane: $(0.5,0.5)$ (point $\mathrm{A}),\left(0.5, b_{c}\right)$ (point B), and $\left(a_{c}, 0.5\right)$ (point C)—see Fig. 5. In Fig. 7 the scaling function is plotted by using the assumption of Eq. 3.3 with appropriate exponent values at point $\mathrm{A},(a, b)=(0.5,0.5)$. It is clear that the scaling assumption is confirmed by the results. Similar results were reported in [17] for points B and C.

Interestingly, while $\mathcal{K}$ is monotonic at points $A$ and $C$, it was found that it distinctly unimodal at point $B$. It was concluded that at points $A$ and $C$, the polymer exerts a repulsive force on the walls at all lengths and widths. Whereas at point $B$ there is a combination of length and width such that the free energy has derivative (with respect to $w$ ) equal to zero. At point $A$ the interactions with both confining walls are 
repulsive and the entropy loss due to confinement leads to a repulsive force. Point $C$ corresponds to a critical value of the attraction at the wall where the walk is tethered and there is no attractive force with the other wall, so the force is repulsive. At point $B$ the walk is tethered to one wall but attracted to the other. If $n \rightarrow \infty$ at fixed $w$ it is known rigorously that the force is repulsive and this corresponds roughly to the case where $n^{v} / w>>1$. If $n^{v}<<w$ the walk extends to allow vertices in the top wall and this leads to an attractive force. The results at point B show new and qualitatively different behaviour from that found in studies where $a=b$ or $b=1$ [3,9-13].

\section{Discussion}

We have reviewed recent work [14-17] on self-avoiding walks confined between walls with which they interact via a contact potential that may be different for the two walls. Such a situation, named the infinite slab, differs from both the case of adsorption of a polymer on one wall and a polymer confined between two non-interacting walls in significant ways. This work $[15,17]$ has conjectured a full phase diagram and also delineated the types of forces between the walls in all regions of the parameter space. These conjectures mimic the exact results found in the two-dimensional integrable model that has been analysed [14]. A scaling theory [17] has also been conjectured. It would be of some interest to derive the corresponding scaling theory in the integrable case [23].

Acknowledgements The authors are indebted to Stu Whittington for his collaboration in the works described here and for his indefatigable enthusiasm and scientific curiosity. The authors thank the Australian Research Council (via its support of MASCOS) and NSERC of Canada for financial support.

\section{References}

1. N. Madras, G. Slade, The Self-Avoiding Walk (Birkhauser, Boston, 1993)

2. D. Napper, Polymeric Stabilisation of Colloidal Dispersions (Academic Press, London, 1983)

3. E.A. DiMarzio, R.J. Rubin, J. Chem. Phys. 55, 4318 (1971)

4. A. Milchev, K. Binder, Eur. Phys. J. B 3, 477 (1998)

5. A. Milchev, A. Bhattacharya, J. Chem. Phys. 117, 5415 (2002)

6. H. Hsu, P. Grassberger, Eur. Phys. J. B 36, 209 (2003)

7. H. Hsu, P. Grassberger, J. Chem. Phys. 120, 2034 (2004)

8. M. Daoud, P.G. de Gennes, J. Phys. 38, 85 (1977)

9. D. Chan, B. Davies, P. Richmond, J. Chem. Soc. Faraday Trans. 72, 1584 (1976)

10. K.M. Middlemiss, G. Torrie, S. Whittington, J. Chem. Phys. 66, 3227 (1977)

11. T. Ishinabe, J. Chem. Phys. 83, 4151 (1985)

12. J.F. Stilck, K.D. Machado, Eur. Phys. J. B 5, 899 (1998)

13. J.F. Stilck, Brazil. J. Phys. 28, 369 (1998)

14. R. Brak, A.L. Owczarek, A. Rechnitzer, S. Whittington, J. Phys. A 38, 4309 (2005)

15. E.J. Janse van Rensburg, E. Orlandini, A.L. Owczarek, A. Rechnitzer, S. Whittington, J. Phys. A 38, L823 (2005)

16. E.J. Janse van Rensburg, E. Orlandini, S. Whittington, J. Phys. A 39, 13869 (2006)

17. R. Martin, E. Orlandini, A.L. Owczarek, A. Rechnitzer, S. Whittington, J. Phys. A 40, 7509 (2007)

18. J.M. Hammersley, G. Torrie, S.G. Whittington, J. Phys. A: Math. Gen. 18, 101 (1982)

19. E.J. Janse van Rensburg, A. Rechnitzer, J. Phys. A 37, 13869 (2004)

20. K. DeBell, T. Lookman, Rev. Mod. Phys. 65, 87 (1993)

21. B. Nienhuis, Phys. Rev. Lett. 49, 1062 (1982) 
22. R. Brak, A.L. Owczarek, A. Rechnitzer, Exact solutions of some lattice polymer models, Submitted to Proceedings of Lattices and Trajectories: A Symposium of Mathematical Chemistry in honour of Ray Kapral and Stu Whittington, 2007

23. A.L. Owczarek, T. Prellberg, A. Rechnitzer, Finite-size scaling functions for directed polymers confined between attracting walls. In preparation (2007) 